\section{Intervention for intermittent distance exotropia with overcorrecting minus lenses}

${ }^{1}$ Division of Orthoptics, University of Liverpool, Liverpool, UK

${ }^{2}$ Department of Ophthalmology, North Cheshire Hospitals NHS Trust, Warrington, UK

Correspondence: FJ Rowe, Division of Orthoptics, University of Liverpool, Thompson Yates Building, Brownlow Hill, Liverpool, Merseyside L69 3GB, UK

Tel: + 441517945732 ;

Fax: +44 1517945781 .

E-mail: rowef@

liverpool.ac.uk

Received: 24 August 2007 Accepted in revised form: 12 November 2007 Published online: 7 December 2007

\section{Abstract}

Aim There are numerous treatment options for intermittent distance exotropia. The aim of this study is to evaluate the use of overcorrecting minus lenses as a primary treatment option for intermittent distance exotropia (IDEX) and determine ocular alignment status after 5 years from commencement of the study.

Methods Prospective nonrandomised longitudinal cohort study in which 21 patients were recruited with a diagnosis of IDEX. Treatment was instigated with the minimum minus lens required to achieve control of the manifest deviation. The strength of lenses was reduced over time while monitoring the results of orthoptic measurements. The results were evaluated by nonparametric (Wilcoxon) and parametric (paired $t$-test) analysis.

Results Thirteen female and eight male patients were recruited with a mean age of 5 years at the start of treatment. There was a significant reduction in angle of deviation after treatment. Overcorrecting lenses did not appear to induce myopia. Twenty-four per cent of the patients had a successful outcome, $28 \%$ had a good outcome, and $33 \%$ required surgery at a later date.

Conclusions Fifty-two per cent of the patients achieved a successful or good outcome with overcorrecting minus lenses alone, and this was maintained for at least 1 year follow-up. Those requiring surgery had been unable to wean out of lenses but had a successful outcome after one procedure. We recommend overcorrecting minus lenses as a primary treatment option for IDEX with the knowledge that surgery, if subsequently required, is safely delayed to an older age without prior loss of binocular vision. Eye (2009) 23, 320-325; doi:10.1038/sj.eye.6703057; published online 7 December 2007
FJ Rowe', CP Noonan², G Freeman² and J DeBell ${ }^{2}$

Keywords: distance exotropia; IDEX; over minus lenses

\section{Introduction}

Intermittent distance exotropia (IDEX) is a deviation characterised by an exophoria at near fixation and manifest exotropia at distance or far distance fixation. There is typically good binocular function with normal fusional vergence and stereoacuity at near fixation. The patient may be noted to close one eye when looking in the distance and the deviation at distance fixation may worsen when tired or day-dreaming. The eyes may also tend to diverge in bright sunlight and the patient may close one eye in such circumstances. ${ }^{1,2}$ The angle of deviation for near fixation may increase on prolonged disruption of fusion or elimination of accommodation. Cases that show such increase for near fixation are generally recorded as simulated distance types.

Indications for treatment include worsening cosmesis with an increase in angle of deviation, decompensation of the deviation, and weak binocular function. ${ }^{3}$ Timing and method of intervention are controversial. ${ }^{4}$ Conservative treatment options include use of prisms, orthoptic exercises, and over minus lenses, whereas invasive treatment options include strabismus surgery and botulinum toxin A injection.

Because of the uncertainty of surgical results, it may be preferable to delay surgery until the child is older, but with the risk of losing binocular function with an increasing angle of deviation. Hence, conservative options have been deemed useful in this 'delay' period. However, prisms are often inappropriate with larger angles of deviation and may interfere with visual acuity. Orthoptic exercises require 
cooperation and an ability to understand the exercise, which is problematic with young children.

Overcorrecting minus lenses provide an alternative conservative treatment as they are cosmetically acceptable, do not hinder visual acuity, and only require sufficient compliance for spectacle wear during daytime. The principle of their use is to stimulate accommodative convergence, which helps to control the divergent angle of deviation. ${ }^{5}$

We sought to prospectively evaluate overcorrecting minus lenses as an independent treatment option for IDEX to determine its efficacy over a follow-up period of up to 5 years.

\section{Materials and methods}

A prospective, nonrandomised, cohort study was undertaken. Patients with a diagnosis of IDEX requiring treatment to prevent loss of binocular function and/or improve cosmesis and angle of deviation were recruited from outpatient strabismus clinics. There were no age restrictions on recruitment to this study. Exclusion criteria included a history of previous surgery, ocular pathology (eg optic nerve hypoplasia), and bestcorrected visual acuity of less than 6/9 Snellens or $0.2 \log$ MAR.

Orthoptic and ophthalmic assessment before, during, and after treatment included uniocular visual acuities at distance (Snellens or logMAR), cover test, ocular motility, binocular function, prism cover test (PCT), AC/A ratio, cycloplegic refraction, and fundus and media evaluation. Binocular function measures included positive and negative fusional vergences using a prism fusion range at near fixation with a peripheral sized target and stereoacuity assessment at near fixation using Frisby or TNO. AC/A ratio was assessed by the gradient method using +3.00 DS lenses with the following calculation:

$$
\mathrm{AC} / \mathrm{A} \text { ratio }=\frac{\text { PCT at } 1 / 3 \mathrm{~m}-\mathrm{PCT} \text { at } 1 / 3 \mathrm{~m} \text { with }+3.00 \mathrm{DS}}{\text { Accommodation effort }(3 \mathrm{D})}
$$

The gradient method could not always be measured at each assessment due to patient cooperation; therefore, the $\mathrm{AC} / \mathrm{A}$ ratio was also calculated for standardisation across visits with the heterophoria method using the calculation:

$$
\begin{aligned}
\mathrm{AC} / \text { A ratio }= & \operatorname{IPD}(\text { in cms. }) \\
& +\frac{\text { near PCT }- \text { distance PCT }}{\text { Accommodation effort }(3 \mathrm{D})}
\end{aligned}
$$

Indications for treatment in our cases included an increase in the angle of deviation of at least 10 prism

\begin{tabular}{|c|c|}
\hline Score & Component \\
\hline \multicolumn{2}{|c|}{ Home control } \\
\hline 0 & Squint/monocular eye closure never noticed \\
\hline 1 & $\begin{array}{l}\text { Squint/monocular eye closure seen occasionally }(<50 \% \\
\text { of time child observed for distance) }\end{array}$ \\
\hline 2 & $\begin{array}{l}\text { Squint/monocular eye closure seen frequently ( }>50 \% \text { of } \\
\text { time child observed for distance) }\end{array}$ \\
\hline 3 & $\begin{array}{l}\text { Squint/monocular eye closure seen for distance and near } \\
\text { fixation }\end{array}$ \\
\hline \multicolumn{2}{|c|}{ Clinic control near } \\
\hline 0 & $\begin{array}{l}\text { Manifest only after cover test and resumes fusion without } \\
\text { the need for blink or refixation }\end{array}$ \\
\hline 1 & Blink or refixate to control after cover test \\
\hline 2 & $\begin{array}{l}\text { Manifest spontaneously or with any form of fusion } \\
\text { disruption without recovery }\end{array}$ \\
\hline \multicolumn{2}{|c|}{ Clinic control distance } \\
\hline 0 & $\begin{array}{l}\text { Manifest only after cover test and resumes fusion without } \\
\text { the need for blink or refixation }\end{array}$ \\
\hline 1 & Blink or refixate to control after cover test \\
\hline 2 & $\begin{array}{l}\text { Manifest spontaneously or with any form of fusion } \\
\text { disruption without recovery }\end{array}$ \\
\hline
\end{tabular}
dioptres (PD) and/or decompensation of the deviation.
Table 1 The Newcastle control score

NCS total $=$ home + clinic near + clinic distance.

A score of 3 or greater was taken to indicate a requirement for treatment intervention.

In addition, the Newcastle control grading score (NCS) was used to grade the extent of IDEX before, during, and after treatment (Table 1 ). ${ }^{6}$ A score of 3 or greater was taken to indicate a requirement for treatment intervention.

The treatment protocol involved the prescription of the minimum minus lenses to reduce the angle of deviation at distance fixation and allow control of the deviation to an exophoria at near and distance fixation. The minus lenses were placed in a trial frame starting at $-1.00 \mathrm{DS}$ strength and working up in -0.50 DS steps until the patient controlled their deviation at near and distance fixation. The control was determined by the presence of a fusional recovery movement evident on cover test at near and distance fixation plus binocular function measures. The strength of the minus lenses at which control was achieved was the prescription given to the patients. An additional requirement was that visual acuity should be maintained at the same level for distance fixation with and without the overcorrecting minus lenses. The patients were required to wear the prescription full-time during waking hours. They were reviewed on a minimum of 3 monthly appointments. At every appointment, the patients were evaluated by orthoptic assessment for their control and by measurement of their angle of deviation both with and without their minus 
lenses prescription for comparison of the status of their deviation. At 6 months after commencement of the treatment, there was an attempt to reduce the prescription by $-0.50 \mathrm{DS}$ if full control of the IDEX was evident. If it was not possible to reduce the prescription without recurrence of the exotropia, the patients were reviewed over a further 6 months and then an attempt was made again to reduce the lenses. The lenses were progressively reduced in $-0.50 \mathrm{DS}$ steps until the glasses could be discontinued.

The results were evaluated at varying time intervals, that is, in the short-term (initial 6-month period of regaining control of IDEX), medium-term (6-24-month period of reducing lenses), and long-term (6-month period after cessation of lenses).

The outcome measure for success was determined as presence of an exophoria at near, distance, and far distance fixation with binocular control at all distances. Statistical evaluation involved nonparametric analysis of independent variables of binocular function, angle of deviation, and AC/A measures. Analysis of parametric variables was undertaken using the paired $t$-test. The study complied with and was undertaken in accordance with the Tenets of Helsinki, and local research ethical approval was obtained.

\section{Results}

Twenty-one patients were recruited to this study during 2000/2001: 13 female and 8 male. Age at commencement of treatment with overcorrecting minus lenses was a mean of 5 years (range 1-9 years, SD 2.2).

Decompensation of the deviation was present at near fixation in 14 patients with reduced binocular function measures and recovery of cover test at near fixation.

Median and/or mean values plus ranges are provided in Table 2 for orthoptic measurements and use of overcorrecting minus lenses.

The median prism fusion range at near fixation to a peripheral sized target was 20 base-out (positive range) to 12 base-in (negative range) compared to an expected norm of 40 base-out to 20 base-in..$^{7}$ Only one patient had a normal prism fusion range at near fixation and this patient had the smallest angle of deviation for near fixation of 4 PD.

In the short-term (initial 6-month period of regaining control of IDEX), the patients were prescribed overcorrecting minus lenses ranging from -1.00 to $-3.00 \mathrm{DS}$ in addition to their baseline prescription (if any). Improvement in angle of deviation and/or binocular function was first noted after a median of

Table 2 Orthoptic measurements and use of overcorrecting minus lenses

\begin{tabular}{lll}
\hline & Median & Mean \\
\hline $\begin{array}{l}\text { Initial measurements } \\
\text { Visual acuity }\end{array}$ & $6 / 6$ Snellen & $6 / 5$ to $6 / 9$ \\
Convergence & $6 \mathrm{~cm}$ & $6-16 \mathrm{~cm}$ (nil in one case) \\
Stereoacuity & $360^{\prime \prime}$ & $75-600^{\prime \prime}$ (nil in two cases) \\
Prism fusion range & 20 base-out & $8-40$ PD (nil in two cases) \\
& 12 base-in & $6-18$ PD (nil in two cases) \\
Alternating prism cover test & -14 PD near & 0 to -25 PD \\
AC/A ratio & -25 PD distance & -16 to -45 PD \\
NCS & $6: 1$ gradient & $2-12: 1$ \\
& $6.6: 1$ heterophoria & $1.6-13.4: 1$ \\
Overcorrecting minus lenses & 4 & $2-5$ \\
Prescription given & & \\
Duration in lenses & & -1.00 to -3.00 DS \\
Improvement noted & -2.00 DS & $6-62$ months \\
NCS (mid-treatment) & 33 months & $1-21$ months \\
Follow-up & 4 months & $0-2$ \\
Last measurements & 1 & $6-39$ months \\
Prism fusion range & 30 months & $-2.00 \mathrm{DS}$ \\
Alternating prism cover test & & \\
AC/A ratio & 20 base-out & $6-45$ PD \\
NCS & 12 base-in & $4-25$ PD \\
\hline
\end{tabular}

$\mathrm{DS}=$ dioptre sphere; $\mathrm{PD}=$ prism dioptres; $"=$ seconds of arc.

Median and mean values plus ranges for initial and last orthoptic measurements including values for use of overcorrecting minus lenses. 
4 months after commencement of treatment. Three patients required an increase in over minus lens prescription during their follow-up. An attempt had been made to reduce their prescription but on follow-up, their binocular control had deteriorated, hence the requirement to increase the prescription again.

In the medium-term (6-24-month period of reducing lenses), binocular vision and thus control of the deviation was evident. The Newcastle control ranged from 0 to 2 with a median of 1 compared to an initial median of 4 (range 2-5) and a final median of 0 (range 0-2).

In the long-term (6-month period after cessation of lenses), the patients were followed for a minimum of 1 year up to $5 \frac{1}{2}$ years (median 2.5 years) after commencement of treatment, and for 6 months up to 39 months after cessation of treatment. There was no significant change in the base-out or base-in prism fusion range over time, $P=0.207$, and $P=0.105$, respectively (Wilcoxon test). Individual base-out and base-in values were combined to produce fusional amplitudes, and a comparison of amplitudes over time revealed no significant change, $P=0.14$ (Wilcoxon test). There was a significant reduction in angle of deviation at both near and distance fixation over time, $P=0.004$ and $P=0.0001$, respectively (Wilcoxon test). There was also a significant reduction in $\mathrm{AC} / \mathrm{A}$ ratio over time, $P=0.006$ (Wilcoxon test).

When analysing baseline refractive errors, we required annual refraction results. Sufficient information on annual repeated refractive error baselines was available only in nine patients, over a median follow-up period of 3 years. Refractive errors containing cylinder powers were converted to spherical equivalent values. These errors were normally distributed in the nine patients. The mean change over the 3-year time period was $-0.62 \pm-0.87 \mathrm{D}$, which was significant, $P=0.025$ (paired $t$-test).

The status of patients in this study, 5 years after commencement of the trial, includes a successful outcome in five patients with full control of the deviation and a good outcome in six patients in whom an occasional manifest deviation is noted at distance fixation, but which is asymptomatic and not a functional or cosmetic problem. Three patients remain in overcorrecting minus lenses and are well controlled in a minimal prescription ( -1.00 DS or less) and seven patients required further treatment, being unable to wean them out of minus lenses without recurrence of a problematic exotropia. All patients requiring further treatment underwent surgery (two patients had diagnostic botulinum toxin at least 6 months prior to surgery) of a unilateral lateral rectus muscle recession and medial rectus muscle resection, or bilateral lateral rectus muscle recessions. All had a successful outcome without recourse to additional treatment.

The patients requiring further treatment were compared to those with a successful or good outcome. Factors such as age when starting treatment, initial angle of deviation, AC/A ratio or NCS, or strength and time period of wearing overcorrecting minus lenses, were not significantly different across the groups.

\section{Discussion}

In the treatment of IDEX, there have been no randomised, controlled trials of treatment options or timing of treatment. IDEX has been considered a progressive strabismus. ${ }^{8}$ Clinical investigations have reported a $23-75 \%$ increase in the angle of deviation, generally over a 5 -year follow-up period..$^{9-12}$ A total of 9-58\% of patients show no change in the angle of deviation and 3.6-34\% of patients show improvement in the angle of deviation. Some observation studies are flawed in that conservative treatment had been offered to patients with subsequent impact on outcome measures over the follow-up period. ${ }^{9,13,14}$ For example, in an early study by Hiles et $a l,{ }^{13} 80 \%$ of subjects had received conservative treatment and the study reported $83 \%$ with no change in the angle over follow-up.

There have been few studies addressing the treatment of IDEX with over minus lenses. Caltrider and Jampolsky ${ }^{5}$ stated that the purpose of nonsurgical treatment was to increase the duration of bifoveal fixation in children, and they conducted their retrospective study to determine if the exo deviation could be improved while wearing over minus lenses and whether this improvement could be maintained when the lenses were discontinued. They discontinued treatment after 6 months if there was a lack of response to the lenses. Their results fell into three groups: (1); $46 \%$ achieved a well-controlled exophoria, (2); $26 \%$ had a reduction in the angle of deviation of 15 PD, and (3); $28 \%$ had no change while using the minus lenses. Out of 10 patients followed for 1 year post-cessation of treatment, 7 maintained their exophoria. They stated that age at start of the treatment was not significant.

More recently, Watts et al ${ }^{15}$ used the Newcastle control score to evaluate the effectiveness of over minus lenses as a treatment option. Seventy-two per cent of their patients showed an improvement, although patients were not followed post-treatment to ascertain maintenance of improvement. They stated that pretreatment angle of deviation, particularly large angles, was not detrimental to a good outcome.

In our prospective study, we had a $100 \%$ improvement while the patients were wearing their over minus lenses in the short-term. In the medium-term, we found that we 
could not reduce the lenses without recurrence of the IDEX, which required further treatment in seven cases. In six further cases, the deviation recurred at distance fixation but at a smaller angle of deviation, which was cosmetically acceptable and with good binocular function at near fixation, thereby requiring no further treatment. Three patients remain in a low minus lens prescription.

In the long-term, five patients achieved exophoria at near and distance fixation with good binocular function, and this was maintained after cessation of treatment.

Our results are similar to other studies ${ }^{5,15}$ in the mediumand long-term. Equally, we did not find AC/A ratio or size of angle of deviation to be detrimental to a good outcome. The mean period of time for use of over minus lenses in one study was $2-156$ months, ${ }^{5}$ which is comparable to our range of use of overcorrecting minus lenses.

The NCS provides guidance on when to consider intervention and has more recently been amended. ${ }^{16}$ It takes into consideration the angle of deviation and the level of control at both near and distance fixation along with consideration of parental observations of the frequency of noticeable strabismus. The score was useful in determining the effect of treatment whilst wearing the overcorrecting minus lenses, and the score values were consistently lower during as well as after cessation of treatment.

A possible contraindication to the use of overcorrecting minus lenses is the possibility that such use could induce the development of actual myopia in the patient. Kushner ${ }^{17}$ compared a group of patients with IDEX undergoing treatment with overcorrecting minus lenses with a group not having this treatment as well as with a further control group. He found a mean change over 5 years of $-1.54 \pm-1.80 \mathrm{D}$ and in comparison of this change to the other groups in the study, he concluded that there was no significant difference in change of refractive error over time and thus no induced myopia from wearing overcorrecting minus lenses. We were able to review refractive change over a median of 3 years and found a mean change in refractive error of $-0.62 \pm-0.87 \mathrm{D}$, which, based on similar changes in Kushner's study, we feel reflects the normal emmetropisation change in refractive error over time for children and thus the unlikely development of myopia due to overcorrecting minus lenses.

Considering the varying results following surgical treatment of IDEX, it is encouraging to have a conservative treatment option from which results appear promising. We have found that the use of overcorrecting minus lenses has attained a successful or good outcome in patients with IDEX in 52\% of the cases in this study. Thirty-three per cent of the cases required further surgery to achieve successful control of their deviation.
We recommend that overcorrecting minus lenses be considered a primary treatment option for IDEX for the following reasons: the deviation is often detected at a young age; the literature reports variable results of surgical treatment in young children with IDEX; success after surgery has been reported as $69 \%$ achieving alignment and $50 \%$ of these achieving alignment and sensory function; ${ }^{3}$ surgery has a reported incidence of overcorrection of $10-15 \%$ and those who have undergone surgery have also been reported to have a subsequent increase in angle of deviation over the postoperative follow-up period. ${ }^{9}$

Use of overcorrecting lenses has the advantage of achieving binocular control of the deviation while wearing the lenses, thus allowing a period of time to consolidate binocular experience and thus promoting good binocular asymptomatic control of the deviation. In $24 \%$ of the cases in our study, the patients were rendered completely controlled after the use of overcorrecting minus lenses while a further $28 \%$ achieved good control, that is only occasional and asymptomatic appearance of a distance exotropia. This is at least comparable to reported surgical results but without the need for a surgical intervention. In the patients that could not be weaned out of minus lenses without recurrence of a problematic exotropia, all had one surgical episode to align the exotropia. This treatment was delayed to a median age of 8 years, at which the children were cooperative and full orthoptic measurements were possible. It is conceivable that these patients obtained excellent control of their deviation following surgery, because of the positive binocular experience they had while wearing the minus lenses preoperatively.

There are a number of limitations to our study. There were a small number of patients recruited to this particular study. We have similarly treated further IDEX patients over more recent years and these appear to follow the same trends as the main study. We were unable to obtain annual refraction information as not all

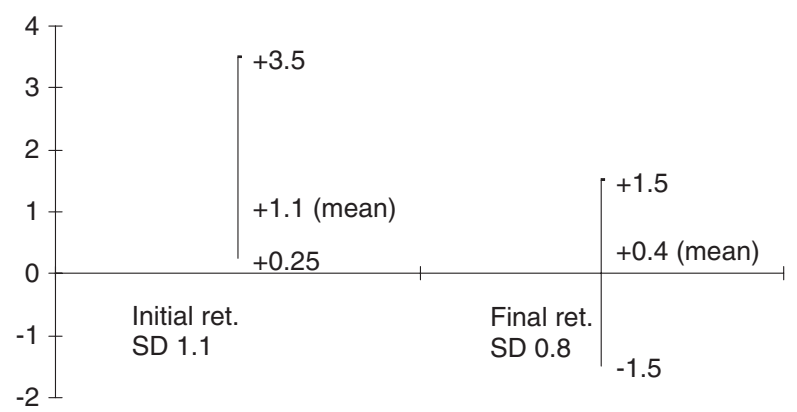

Figure 1 Mean change in refractive error. The mean plus upper and lower limits of refractive errors are shown for initial and final baseline refractions. 
children had their refractions undertaken within our hospital department. This curtailed our evaluation of refractive change to nine patients. However, there were normally distributed data and the mean change over time appeared consistent with the literature (Figure 1).

This has been a clinical study involving patients from the age of 1-year-old. Therefore, there have been limitations of patient responses during testing related to cooperation. Although we have had good compliance with spectacle wear, we acknowledge that this can be problematic with some children and this must be considered in wider clinical practice. However, we still believe that it is worth promoting a trial of overcorrecting minus lenses to determine their effect on the strabismic angle.

\section{References}

1 Holt R. A study of divergent strabismus in Australia. Br Orthoptic J 1951; 8: 95.

2 Berg PH. Effect of light intensity on the prevalence of exotropia in strabismus populations. Br Orthoptic Journal 1982; 39: 55

3 Yildirim C, Mutlu FM, Chen Y, Altinsoy HI. Assessment of central and peripheral fusion and near and distance stereoacuity in intermittent exotropic patients before and after strabismus surgery. Am J Ophthalmol 1999; 128: 222-230.

4 Richardson SR, Gnanaraj L. Interventions for intermittent distance exotropia. Cochrane Database Syst Rev 2003, Issue 2. Art. No.: CD003737.

5 Caltrider N, Jampolsky A. Overcorrecting minus lens therapy for treatment of intermittent exotropia. Ophthalmology 1983; 90: 1160-1165.
6 Haggerty H, Richardson S, Hrisos S, Strong NP, Clarke MP. The Newcastle control score: a new method of grading the severity of intermittent distance exotropia. $\mathrm{Br} J \mathrm{Ophthalmol}$ 2004; 88: 233-235.

7 Tait EF. Fusional vergence. Am J Ophthalmol 1949; 32: 1223-1230.

8 Rosenbaum A, Strathocopoulos R. Subjective and objective criteria for recommending surgery in intermittent exotropia. Am Orthopt J 1992; 42: 46-51.

9 Nusz KJ, Mohney BG, Diehl NN. The course of intermittent exotropia in a population-based cohort. Ophthalmology 2006; 113: $1154-1158$.

10 Romanchuk KG, Dotchin SA, Zurevinsky J. The natural history of surgically untreated intermittent exotropia-looking into the distant future. J AAPOS 2006; 10: 225-231.

11 Chia A, Seenyen L, Long QB. A retrospective review of 287 consecutive children in Singapore presenting with intermittent exotropia. J AAPOS 2005; 9: 257-263.

12 Rutstein RP, Corliss DA. The clinical course of intermittent exotropia. Optom VisSci 2003; 80: 644-649.

13 Hiles D, Davies G, Costenbader F. Long-term observations on unoperated intermittent exotropia. Arch Ophthalmol 1968; 80: $436-442$.

14 Sanfillippo S, Clahane A. The effectiveness of orthoptics alone in selected cases of exodeviations: the immediate results and several years later. Am Orthopt J 1970; 20: 104-117.

15 Watts $\mathrm{P}$, Tippings E, Al-Madfai H. Intermittent exotropia, overcorrecting minus lenses and the Newcastle scoring system. JAAPOS 2005; 9: 460-464.

16 Clarke MP. Intermittent exotropia. J Pediatr Ophthalmol Strabismus 2007; 44: 153-157.

17 Kushner BJ. Does overcorrecting minus lens therapy for intermittent exotropia cause myopia? Arch Ophthalmol 1999; 117: 638 . 